\title{
Involvement of p53 R72P polymorphism in the association of MDM2-SNP309 with breast cancer
}

\author{
JYH-DER LEU ${ }^{1,6^{*}}$, CHUNG-YIH WANG ${ }^{2 *}$, HAN-YI TSAI ${ }^{3}$, \\ I-FENG LIN ${ }^{4}$, RAN-CHOU CHEN ${ }^{3,5}$ and YI-JANG LEE ${ }^{1,3}$
}

\begin{abstract}
${ }^{1}$ Division of Radiation Oncology, Taipei City Hospital Renai Branch, Taipei 106; ${ }^{2}$ Division of Radiation Oncology, Cheng-Hsin General Hospital, Taipei 106; ${ }^{3}$ Department of Biomedical Imaging and Radiological Sciences, National Yang-Ming University, Taipei 112; ${ }^{4}$ Department of Public Health, National Yang-Ming University, Taipei 112; ${ }^{5}$ Department of Radiology, Taipei City Hospital, Taipei 106; ${ }^{6}$ Institute of Neuroscience, National Chengchi University, Taiwan, R.O.C.
\end{abstract}

Received January 20, 2011; Accepted March 15, 2011

DOI: $10.3892 /$ or.2011.1254

\begin{abstract}
The allelic variant MDM2-SNP309 (T>G) has been suggested to influence cancer development, but the clinical correlation between the risk allele and breast cancer remains controversial. The genetic background and the ethnicity of selected subgroups may influence the power of these risk genotypes. In this study, we investigated whether MDM2-SNP309 is associated with p53 R72P genetic polymorphism for the risk of breast cancer development in Asian Taiwanese, which has not been well-studied in this regard. Two hundred and fifty-five patients and 324 cancer-free controls were included, and we found that the MDM2-SNP309 TG and GG genotypes displayed marginally increased risks of breast cancer (GG vs. TT: $\mathrm{OR}=1.7,95 \% \mathrm{CI}=0.93$ to 3.09 ; $\mathrm{TG}+\mathrm{TT}$ vs. TT: $\mathrm{OR}=1.57,95 \% \mathrm{CI}=0.98$ to 2.56 ). The breast cancer risk asso-ciated with MDM2-SNP309 was enhanced after stratification for the homozygous GG genotype at $\mathrm{p} 53$ codon 72 representing the Arg form of this genotype (GG vs. TT: OR $=3.7,95 \% \mathrm{CI}=1.144$ to $12.02 ; \mathrm{TG}+\mathrm{GG}$ vs. $\mathrm{TT}: \mathrm{OR}=2.7$, $95 \% \mathrm{CI}=1.027$ to 6.895$)$. Also, the median age at diagnosis of patients with MDM2-SNP309 GG increased from 4 years earlier to 9 years earlier than TT patients after stratification for the GG geno-type at p53 codon 72. Moreover, the G-allele of MDM2-SNP309 exhibited a stronger capacity than the T-allele to drive the full-length P2 promoter of the MDM2 gene in several human cell lines, suggesting that the association between MDM2-SNP309 and breast cancer is likely
\end{abstract}

Correspondence to: Dr Yi-Jang Lee, Department of Biomedical Imaging and Radiological Sciences, National Yang-Ming University, No. 155, Sec. 2, Linong St. Beitou District, Taipei 112, Taiwan, R.O.C.

E-mail: yjlee2@ym.edu.tw

*Contributed equally

Key words: MDM2-SNP309, p53 codon R72P, breast cancer risk, Asian Taiwanese multifactorial rather than due to inconsistent gene expression in different cancer sources.

\section{Introduction}

According to the World Health Organization (WHO), breast cancer remains the highest mortality-and morbidity-associated female cancer in both developing and developed countries. It may be associated with a high-calorie diet, environmental and career stresses, and/or other social-physiological factors. Given that cancer is considered to be a genetic disease, genetic polymorphisms may also affect the risk of breast cancer $(1,2)$. Although rare, the incidence of male breast cancer is increasing because of hormonal, environmental and genetic risk factors (3).

The p53-MDM2 circuit is an important auto-regulatory mechanism that mediates responses to various cellular and environmental stresses that include cell cycle arrest, apoptosis, senescence and DNA repair (4). Mutations in p53 are associated with $50 \%$ of human cancers. With regard to breast cancer, the genomic mutation of the p53 gene or the nuclear accumulation of impaired p53 accounts for $20-40 \%$ of all cases at different disease stages $(5,6)$. In addition, the repression of p53 activation by the estrogen-mediated over-expression of MDM2 enhances the incidence rate of breast cancer $(7,8)$. This enhancement is associated with a single nucleotide polymorphism found in the P2 promoter region of the MDM2 gene, SNP309 (T to $\mathrm{G}$ change; rs2279744). The homozygous MDM2-SNP309 genotype (GG) is known to increase the basal level of MDM2 protein by enhancing the binding of the Sp1 general transcription factor to the promoter (9). This may explain why young women harboring MDM2-SNP309 are prone to breast cancer because high estrogen secretion usually occurs in adolescence for the maintenance of sexual characteristics (10).

The association between MDM2-SNP309 and the risk of breast cancer remains controversial. For example, a large pooled series of $>5,000$ cases from five European studies within the Breast Cancer Association Consortium (BCAC) concluded that no association can be found between MDM2-SNP309 and breast cancer (11). By contrast, we and others have 
found that MDM2-SNP309 remains a risk factor for breast cancer in various patient subgroups (12-15). Using a metaanalysis, Economopoulos and Sergentanis focused on the racial effect and proposed that MDM2-SNP309 affects the incidence of breast cancer in the Chinese population but not the non-Chinese population (16). Whether the effects of MDM2-SNP309 on breast cancer incidence are associated with the racial factor alone or the genetic backgrounds of different races requires further investigation.

The germ-line variant of p53 codon $\mathrm{R} 72 \mathrm{P}$ is due to a $\mathrm{G}$ to $\mathrm{C}$ nucleotide change at codon 72 located in exon 4 (rs1042522) that changes the codon from Arg (R) to Pro (P) (17). The $72 \mathrm{R}$ variant tends to activate the apoptotic pathway, whereas the $72 \mathrm{P}$ variant promotes cell cycle arrest and DNA repair in stressed cells (18-21). The association between p53 codon $\mathrm{R} 72 \mathrm{P}$ and the risk of breast cancer is not significant according to an international large cohort study in Europe $(1,11)$. A recent meta-analysis of 39 case-control studies (26,041 cancer cases and 29,679 controls) showed that p53 codon R72P may decrease the risk of breast cancer, especially in Europeans (22). On the other hand, a small population study from Greece concluded that p53 Arg homozygosity is a risk factor for breast cancer (23). These different conclusions from the same population suggest that the association between p53 codon $\mathrm{R} 72 \mathrm{P}$ and breast cancer is complicated and may depend on the selected subgroups.

Over-expression of MDM2 leads to the down-regulation of p53 and results in a higher risk of carcinogenesis and accelerated tumorigenesis (24). Homozygous MDM2-SNP309 has been reported to increase the risk of several human cancers harboring wild-type p53 $(7,15,25,26)$. Several reports have focused on the combined effects of MDM2-SNP309 and p53 codon $\mathrm{R} 72 \mathrm{P}$ on different human cancers, and the risk association is controversial (27-32). The interaction between MDM2-SNP309 and p53 codon R72P is not associated with breast cancer risk, although it may affect the age at onset and patient's survival (11,33-36). Because the studied populations in these reports are mainly from Western countries and India, it is difficult to exclude the combined effects of these two genetic variants on the risk and development of breast cancer in different races or subgroups.

We have previously reported that MDM2-SNP309 increases the risk of breast cancer and advances its age of onset in Asian-Taiwanese women (12). Little is known about whether p53 codon R72P interacts with MDM2-SNP309 during the development of breast cancer. The effects of p53 codon R72P on the risk of breast cancer are also not well-studied. Because these combined genetic variants may influence the risk of human cancer differently in different subgroups, we investigated the interaction of MDM2-SNP309 and p53 codon R72P during breast cancer development in the Asian-Taiwanese population.

\section{Materials and methods}

Patient samples. Two hundred and fifty-five breast cancer patients and 324 cancer-free healthy patients were collected from Taipei City Hospital Ren-Ai Branch in Taipei, Taiwan. The patients were consecutively and randomly enrolled. The participants were guided to sign consent forms and structured questionnaires after they had agreed to the research content. The demography of these patients is summarized in Table I. The participants were all Asian Taiwanese, not immigrants from America or Europe. This study was approved by the Taipei City Hospital Institutional Review Board (TCHIRB-970705).

Genotyping of MDM2 SNP309 and p53 codon R72P. For genotyping, $3 \mathrm{ml}$ of whole blood was collected from each participant. The preparation of genomic DNA from whole blood samples was carried out, and the MDM2-SNP309 genotyping procedure was performed as previously described with minor modifications (37). In brief, using the Qiagen mini blood DNA extraction kit (Qiagen Inc., Valencia, CA), genomic DNA was extracted from $200 \mu \mathrm{l}$ of blood and stored at $-20^{\circ} \mathrm{C}$ until use. The chromosomal region containing MDM2 SNP 309 was amplified by polymerase chain reaction (PCR) using a pair of primers, namely F (5'-CGGGAGTTCAGGGTAAAGGT-3') and R (5'-AGCAAGTCGGTGCTTACCTG-3'). The PCR reactions consisted of $100 \mathrm{ng}$ of genomic DNA, $0.2 \mu \mathrm{M}$ primer, $200 \mu \mathrm{M}$ dNTP, $1.5 \mathrm{mM} \mathrm{MgCl}_{2}, 20 \mathrm{mM}$ Tris- $\mathrm{HCl}(\mathrm{pH} 8.4)$, $50 \mathrm{mM} \mathrm{KCl}$ and $1 \mathrm{U}$ of Platinum Taq DNA polymerase (Invitrogen, Carlsbad, CA). The thermal cycling conditions were $1 \mathrm{~min}$ at $94^{\circ} \mathrm{C} ; 40$ cycles of denaturing at $94^{\circ} \mathrm{C}$, annealing at $58^{\circ} \mathrm{C}$, and elongation at $72^{\circ} \mathrm{C}$ for $30 \mathrm{sec}$ each, followed by one cycle at $72^{\circ} \mathrm{C}$ for $10 \mathrm{~min}$. For restriction fragment length polymorphism (RFLP) analysis, 10-20 $\mu \mathrm{l}$ of the amplified 352-bp fragment was digested with $1 \mathrm{U}$ of MspA1I restriction enzyme (New England Biolabs, Ipswich, MA) at $37^{\circ} \mathrm{C}$ in a water bath for $30 \mathrm{~min}$ to 1 hour. The T/T, T/G and G/G genotypes were distinguished by observing the presence of bands with lengths of 233 and 88; 233, 187 and $88 \mathrm{bp}$; and 187 and $88 \mathrm{bp}$, respectively, after electrophoresis on a 3\% NuSieve agarose gel. For p53 codon 72 polymorphism genotyping, the procedure was followed as described previously (38). The primer set was: F (5'-TTTCACCCATCTACAGTCCC-3') and R (5'-CGGTGTAGGAGCTGCTG-3'). The length of the PCR product was $166 \mathrm{bp}$, and this was then digested with BstU1 at $60^{\circ} \mathrm{C}$ for $1 \mathrm{~h}$. The digestion of the $\mathrm{P} / \mathrm{P}$ variant yielded a 166-bp band, the R/R variant yielded 135- and 31-bp bands, and the $\mathrm{P} / \mathrm{R}$ heterozygous variant yielded 166, 135 and $31 \mathrm{bp}$ bands. In addition, the genotypes were further confirmed by direct sequencing of the PCR products by the Sequencing Core Facility of the National Yang-Ming University Genomic Research Center (YMGC).

Cell culture. Human breast cancer cell lines (including MDAMB-231, MDA-MB-435s, BT474 and MCF-7) and human embryonic kidney 293T cells were cultured in Dulbecco's modified Eagle's medium (DMEM) supplemented with $10 \%$ fetal bovine serum (FBS, Sigma), 2 mM L-glutamate, $50 \mathrm{U} / \mathrm{ml}$ penicillin and $50 \mu \mathrm{g} / \mathrm{ml}$ streptomycin (Invitrogen). For BT474 cells, $10 \mu \mathrm{g}$ of non-essential amino acids was also supplemented. All cell lines were maintained in a humidified $37^{\circ} \mathrm{C}$ incubator supplemented with $5 \% \mathrm{CO}_{2}$ and $95 \%$ air. BT474 cells were sub-cultured every four days, whereas all other cell lines were passaged every two days.

Plasmid constructs. Genomic PCR was used to obtain the P2 promoter region of the MDM2 gene. The sequences of the primers were F: 5'-CTCGAATTCGGAGCAGGTACTGGC 
Table I. The demography of breast cancer patients and cancer-free controls from blood donors.

\begin{tabular}{|c|c|c|c|c|c|c|}
\hline \multirow[b]{2}{*}{ Characteristics } & \multicolumn{3}{|c|}{ MDM2-SNP309a } & \multicolumn{3}{|c|}{ p53 R72P } \\
\hline & Cases & Controls & p-value & Cases & Controls & p-value \\
\hline \multicolumn{7}{|l|}{ Age (years) } \\
\hline Mean & 55.2 & 62.1 & \multirow[t]{2}{*}{$<0.0001$} & 55.0 & 62.3 & \multirow[t]{2}{*}{$<0.0001$} \\
\hline $\mathrm{SD}$ & 10.7 & 19.6 & & 10.8 & 19.6 & \\
\hline \multicolumn{7}{|l|}{ Gender } \\
\hline Male & $1 \quad(0.4)$ & $132(40.7)$ & \multirow[t]{2}{*}{$<0.0001$} & $1 \quad(0.4)$ & $132(41.1)$ & \multirow[t]{2}{*}{$<0.0001$} \\
\hline Female & 254 (99.6) & $192(59.3)$ & & $238(99.6)$ & $189(58.9)$ & \\
\hline \multicolumn{7}{|l|}{ Alcoholism } \\
\hline Yes & $15(5.9)$ & $35(10.8)$ & \multirow[t]{2}{*}{0.052} & $17(7.1)$ & $33(10.3)$ & \multirow[t]{2}{*}{0.25} \\
\hline No & $240(94.1)$ & $289(89.2)$ & & $222(92.9)$ & $288(89.7)$ & \\
\hline \multicolumn{7}{|c|}{ High calorie intake } \\
\hline Yes & $55(21.6)$ & $73(22.5)$ & \multirow[t]{2}{*}{0.86} & $50(20.9)$ & $72(22.4)$ & \multirow[t]{2}{*}{0.75} \\
\hline No & $200(78.4)$ & $251(77.5)$ & & $189(79.1)$ & $249(77.6)$ & \\
\hline \multicolumn{7}{|l|}{ Vegetarian } \\
\hline Yes & $18(7.1)$ & 10 & \multirow[t]{2}{*}{0.044} & $16(6.7)$ & $10(3.1)$ & \multirow[t]{2}{*}{0.074} \\
\hline No & 237 (92.9) & $314(96.9)$ & & $223(93.3)$ & $311(96.9)$ & \\
\hline \multicolumn{7}{|c|}{ Cardiovascular disorders } \\
\hline Yes & $72(28.2)$ & $141(43.5)$ & \multirow[t]{2}{*}{0.0002} & $67 \quad(39)$ & $140(43.6)$ & \multirow[t]{2}{*}{0.0002} \\
\hline No & $183(71.8)$ & $183(56.5)$ & & $172 \quad(61)$ & $181(56.4)$ & \\
\hline \multicolumn{7}{|c|}{ Body mass index (BMI) } \\
\hline Mean & 23.9 & 23.6 & \multirow[t]{2}{*}{0.24} & 24.0 & 23.60 & \multirow[t]{2}{*}{0.1307} \\
\hline SD & 3.9 & 3.4 & & 3.9 & 3.44 & \\
\hline
\end{tabular}

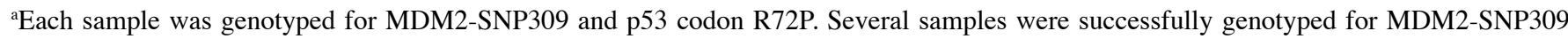

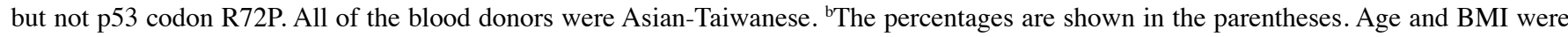
determined by two-sample $t$ tests, and all other parameters were determined by chi-square tests.

CCGGC-3' and R: 5'-GACAAGCTTTCGCCACTGAACA CAGCTGG-3'. The genomic DNA extracted from MCF-7 cells (harbors heterozygous MDM2-SNP309 (T/G) genotype) was subjected to genomic PCR using the above primers. An approximately 500-bp PCR product was purified by a gel extraction kit (Qiagen) and was cloned into the pGluc-Basic plasmid (Promega, Madison, WI) through EcoRI and HindIII enzyme sites. The obtained clones were collected for plasmid extraction and were all subsequently subjected to DNA sequencing. The constructs containing T or G at the MDM2-SNP309 position were named SNP309T-Gluc and SNP309G-Gluc, respectively.

Transfection and reporter gene assay. Cells were transfected with either the SNP309T or SNP309G reporter gene construct using the jetPEI polymer-based DNA transfection reagent (PolyPlus transfection $^{\mathrm{TM}}$, New York, NY) according to the manufacturer's instruction. After $48 \mathrm{~h}$ of transfection, $50 \mu \mathrm{l}$ of culture medium was collected and mixed with $100 \mu \mathrm{l}$ of coelenterazine substrate (Gold Biotechnology Inc., St. Louis, MO) in 1X luciferase buffer (50 mM glycylglycin, $1 \mathrm{M}$ magnesium sulfate, $10 \mathrm{mg} / \mathrm{ml}$ bovine serum albumin and $0.5 \mathrm{M}$ EDTA). The bioluminescent reaction was immediately measured using the Wallac Victor 2 Multi-label Counter (Perkin Elmer
Inc., Waltham, MA) by detecting the emitted photons at $475 \mathrm{~nm}$. The data were normalized by total protein in $50 \mu \mathrm{l}$ of culture medium. The values are the mean of three independent experiments \pm SD The differences between the values obtained with the two different reporter gene constructs were considered significant at $\mathrm{p}<0.05$.

Statistical analysis. The genotype and allele frequencies were tested for Hardy-Weinberg equilibrium using a publicly available calculator (http://www.genes.org.uk/software/hardyweinberg.shtml), and the statistical significance was determined by the $\chi^{2}$ test. The values of the odd ratios (ORs) and $95 \%$ confidence intervals (CIs) were used to determine the risk association between MDM2-SNP309 genotype, p53 codon $\mathrm{R} 72 \mathrm{P}$ and breast cancer using multivariate logistical regression analysis. The Kaplan-Meier method and the log-rank test were used to compare the breast cancer patients with different MDM2-SNP309 and p53 codon R72P genotypes with respect to age at diagnosis, as described previously (12). The initial date were counted from the date of hospital admission. Statistical differences were considered to be significant at $\mathrm{p}<0.05$. All tests and statistical evaluations were performed by the Statistical Analysis System software (ver. 9.1; SAS Institute, Cary, NC). 
Table II. The risk evaluation of MDM2 SNP309 and p53 R72 R genotypic frequencies on the development of breast cancers in the Taiwanese population.

\begin{tabular}{|c|c|c|c|c|c|c|}
\hline Genotypes & Cases n (\%) & Controls n (\%) & OR $(95 \% \mathrm{CI})^{\mathrm{a}}$ & p-value & OR $(95 \% \mathrm{CI})^{\mathrm{b}}$ & $\mathrm{p}$-value \\
\hline \multicolumn{7}{|c|}{ MDM2-SNP309 } \\
\hline $\mathrm{TT}$ & 47 (18.4) & $90(27.8)$ & 1 (reference) & & 1 (reference) & \\
\hline TG & $150(58.8)$ & $172(53.1)$ & $1.67(1.1-2.53)$ & 0.02 & $1.55(0.94-2.55)$ & 0.08 \\
\hline GG & $58(22.4)$ & $62(19.8)$ & $1.79(1.09-2.96)$ & 0.02 & $1.7 \quad(0.93-3.09)$ & 0.08 \\
\hline $\mathrm{TG}+\mathrm{GG}$ & 208 (81.6) & 234 (72.2) & $1.7 \quad(1.14-2.54)$ & 0.01 & $1.57(0.98-2.56)$ & 0.06 \\
\hline \multicolumn{7}{|c|}{ Allele Frequency ${ }^{\mathrm{d}}$} \\
\hline $\mathrm{T}$ & 0.48 & 0.54 & & & & \\
\hline G & 0.52 & 0.46 & & & & \\
\hline \multicolumn{7}{|c|}{ p53 codon72 R/P } \\
\hline $\mathrm{RR}(\mathrm{GG})^{\mathrm{c}}$ & $71(29.7)$ & 104 (32.4) & 1 (reference) & & 1 (reference) & \\
\hline PR (GC) & $90(37.7)$ & $129(40.2)$ & $1.02(0.68-1.53)$ & 0.9 & $1.03(0.64-1.68)$ & 0.89 \\
\hline $\mathrm{PP}(\mathrm{CC})$ & 78 (32.6) & $88(27.4)$ & $1.22(0.75-1.98)$ & 0.2 & $1.1 \quad(0.66-1.81)$ & 0.71 \\
\hline$P P+P R$ & $168(70.3)$ & 217 (67.6) & $0.90(0.59-1.38)$ & 0.5 & $1.06(0.69-1.64)$ & 0.78 \\
\hline \multicolumn{7}{|c|}{ Allele Frequency } \\
\hline $\mathrm{T}$ & 0.49 & 0.52 & & & & \\
\hline $\mathrm{G}$ & 0.51 & 0.48 & & & & \\
\hline
\end{tabular}

${ }^{\mathrm{a}} \mathrm{Crude}$ OR. ${ }^{\mathrm{b}} \mathrm{ORs}$ are adjusted for age, gender, high calorie intake, vegetarian, and cardiovascular diseases. ${ }^{\mathrm{c}}$ Genotype of the SNP is shown in the parenthesis. ${ }^{\mathrm{d}}$ The $\chi^{2}$ of cases and controls in MDM2-SNP309 group are 8.07 and 1.48, respectively. ${ }^{\mathrm{e}}$ The $\chi^{2}$ of cases and controls in p53 codon R72P are 14.5 and 12.1 , respectively.

\section{Results}

The characteristics of breast cancer patients and cancerfree healthy controls. In this study, we have genotyped the MDM2-SNP309 and p53 codon R72P in 579 subjects (255 cancer cases and 324 controls). The characteristics of the patients and the cancer-free controls are summarized in Table I. Among them, 19 blood samples (16 cancer cases and 3 controls) failed to obtain p53 codon R72P genotypes after several experimental repeats. However, the statistical analysis revealed no significant discrepancy between these two groups for any of the characters. The variants exhibited significant difference $(\mathrm{p}<0.05)$ between patient cases and cancer-free controls were adjusted for the ORs in the multivariate logistic regression analysis.

The frequency of MDM2-SNP309 and of p53 codon R72P in the Taiwanese population and their association with the risk of breast cancer. In the MDM2-SNP309 group, the frequency of the homozygous (GG) and heterozygous (TG) genotypes combined was higher in the patients than in the cancer-free controls (81.6 vs. $72.2 \%$ ). According to the individual genotyping data, the patients also had higher $\mathrm{GG}$ and $\mathrm{TG}$ frequencies than the cancer-free controls (GG: 22.4 vs. $19.8 \%$; TG: 58.8 vs. 53.1\%). In addition, the frequency of MDM2-SNP309 in the cancer-free controls obeyed Hardy-Weinberg equilibrium $\left(\chi^{2}=1.48\right)$, whereas this result was not observed in the cases group $\left(\chi^{2}=8.07\right)$ (Table II). By contrast, only the homozygous CC genotype exhibited higher frequency in the cases $(32.6 \%)$ than in the cancer-free controls $(27.4 \%)$ in the p53 codon $\mathrm{R} 72 \mathrm{P}$ group. The frequency of the p53 codon R72P genotype did not conform to Hardy-Weinberg equilibrium in either the cases or the cancer-free controls (Table II).

The association of MDM2-SNP309 and p53 codon R72P with the risk of breast cancer in the Taiwanese population was further dissected by the OR with a $95 \%$ confidential interval (CI) using multivariate logistic regression analysis. As shown in Table II, the heterozygous and homozygous MDM2-SNP309 genotypes exhibited elevated risk compared to the common genotype (TG: $\mathrm{OR}=1.55,95 \% \mathrm{CI}=0.94$ to 2.55 ; GG: $\mathrm{OR}$ $=1.7,95 \% \mathrm{CI}=0.93$ to 3.09 ). By contrast, the $\mathrm{GC}$ and $\mathrm{CC}$ genotypes of $\mathrm{p} 53$ codon $\mathrm{R} 72 \mathrm{P}$ did not confer a significantly greater risk of breast cancer using the GG genotype as a reference $(\mathrm{GC}: \mathrm{OR}=1.03,95 \% \mathrm{CI}=0.64$ to $1.68 ; \mathrm{CC}: \mathrm{OR}=$ $1.1,95 \% \mathrm{CI}=0.66$ to 1.81 ). The ORs were adjusted for several confounding factors, including age, gender, high caloric intake, vegetarianism and cardiovascular diseases. Thus, it appears that MDM2-SNP309, but not p53 codon R72P in the same studied subject, is associated with the risk of breast cancer in the Taiwanese population. Since the genotypic distribution of p53 codon R72P in both patient cases and cancer-free controls do not conform to the Hardy-Weinberg law, the risk association of this genotype with breast cancer remains to be confirmed.

Evaluation of the impact of MDM2-SNP309 on the risk of breast cancer by stratification with p53 codon R72P. Because MDM2-SNP309 increased the risk of breast cancer in the Taiwanese population, we next investigated whether p53 codon R72P influenced the effects of MDM2-SNP309 on breast cancer incidence. Using the multivariate logistic regression model, MDM2-SNP309 genotypes were stratified for p53 codon 
Table III. The risk evaluation of MDM2-SNP309 on breast cancer stratified for p53 R72P.

\begin{tabular}{|c|c|c|c|c|}
\hline & Cases n (\%) & Controls n $(\%)$ & OR $(95 \% \mathrm{CI})^{\mathrm{b}}$ & p-value \\
\hline \multicolumn{5}{|c|}{ GG genotype of p53 R72Pa } \\
\hline \multicolumn{5}{|c|}{ MDM2-SNP309 } \\
\hline $\mathrm{TT}$ & $11(16.2)$ & $37(35.9)$ & 1 (reference) & \\
\hline TG & $38(55.9)$ & $48(46.6)$ & $2.3(0.886-6.249)$ & 0.02 \\
\hline GG & $19(27.9)$ & $18(17.5)$ & $3.7(1.144-12.02)$ & 0.01 \\
\hline $\mathrm{TG}+\mathrm{GG}$ & $57(83.8)$ & $66(64.1)$ & $2.7(1.027-6.895)$ & 0.005 \\
\hline \multicolumn{5}{|c|}{ Allele frequency } \\
\hline $\mathrm{T}$ & 0.44 & 0.59 & & \\
\hline $\mathrm{G}$ & 0.56 & 0.41 & & \\
\hline \multicolumn{5}{|c|}{ GC genotype of p53 R72P } \\
\hline \multicolumn{5}{|c|}{ MDM2-SNP309 } \\
\hline $\mathrm{TT}$ & $18(21.4)$ & $33(26.2)$ & 1 (reference) & \\
\hline $\mathrm{TG}$ & $51(60.7)$ & $72(57.1)$ & $1.17(0.508-2.702)$ & 0.50 \\
\hline GG & $15(17.9)$ & $21(16.7)$ & $1.47 \quad(0.491-4.4)$ & 0.66 \\
\hline $\mathrm{TG}+\mathrm{GG}$ & $66(78.6)$ & $93(73.8)$ & $1.24 \quad(0.553-2.76)$ & 0.51 \\
\hline \multicolumn{5}{|c|}{ Allele frequency } \\
\hline $\mathrm{T}$ & 0.52 & 0.55 & & \\
\hline $\mathrm{G}$ & 0.48 & 0.45 & & \\
\hline \multicolumn{5}{|c|}{ CC genotype of p53 R72P } \\
\hline \multicolumn{5}{|c|}{ MDM2-SNP309 } \\
\hline $\mathrm{TT}$ & $16(21.6)$ & $18(20.9)$ & 1 (reference) & \\
\hline $\mathrm{TG}$ & $43(58.1)$ & $47(54.7)$ & $0.87(0.335-2.266)$ & 0.99 \\
\hline GG & $15(20.3)$ & $21(24.4)$ & $0.66(0.219-1.964)$ & 0.81 \\
\hline $\mathrm{TG}+\mathrm{GG}$ & $58(78.4)$ & $68(79.1)$ & $0.8 \quad(0.318-1.996)$ & 0.99 \\
\hline \multicolumn{5}{|c|}{ Allele frequency } \\
\hline $\mathrm{T}$ & 0.51 & 0.48 & & \\
\hline $\mathrm{G}$ & 0.49 & 0.52 & & \\
\hline
\end{tabular}

${ }^{a}$ Three genotypes of p53 R72P separately grouped for stratified analysis of the association of MDM2-SNP309 genotypes and breast cancer incidence. ${ }^{b}$ Each OR has been adjusted for age, gender, high calorie intake, vegetarian and cardiovascular diseases using the multivariate logistic analysis. 'The p-value was determined by Fisher's exact test. ${ }^{\mathrm{d}}$ The allelic frequency was obtained by the Hardy-Weinberg equilibrium, and the MDM2-SNP309 genotype in each stratified group conforms to the equilibrium.

R72P with adjustment. The results showed that the p53 codon R72P GG genotype (which encodes RR) enhanced the effects of both the heterozygous and homozygous MDM2-SNP309 genotypes on the risk of breast cancer (TG: $\mathrm{OR}=2.3,95 \%$ $\mathrm{CI}=0.886$ to 6.249 ; $\mathrm{GG}: \mathrm{OR}=3.7,95 \% \mathrm{CI}=1.144$ to 12.02 ) (Table III). On the other hand, the p53 codon R72P CC genotype (encodes PP) negatively influenced the risk of MDM2-SNP309 on breast cancer (TG: $\mathrm{OR}=0.87,95 \% \mathrm{CI}=0.335$ to 2.266 ; GG: $\mathrm{OR}=0.66,95 \% \mathrm{CI}=0.219$ to 1.964$)$. The distribution of MDM2-SNP309 genotypes obeys Hardy-Weinberg equilibrium in both cases and controls after they were stratified for p53 codon $\mathrm{R} 72 \mathrm{P}$.

Effects of MDM2-SNP309 and p53 codon R72P genotypes on age at onset of breast cancer. We next compared the median age at diagnosis of breast cancer cases between the different genotypes of MDM2-SNP309. For MDM2-SNP309, the median ages of onset for the GG and TT genotypes were 50 (range 37-72) and 54 (range 34-91) years, respectively.
In patients bearing the p53 codon R72P GG genotype, the median age at diagnosis for the MDM2-SNP309 GG and TT genotypes was 50 and 59 years, respectively. This significant early age onset of homozygous MDM2-SNP309 genotype was not observed under other genotype background of p53 codon R72P (R72P GC genotype, SNP309GG vs TT: 49 vs 53 years; R72P CC genotype, SNP309GG vs TT: 55 vs 53 years). The age at onset of breast cancer with different combinations of MDM2-SNP309 and p53 codon R72P genotypes was also analyzed by the Kaplan-Meier method with the log-rank test. The results showed that despite significant differences in the onset ages between the homozygous and common genotypes of MDM2-SNP309 and p53 codon R72P were not observed, the homozygous MDM2-SNP309 genotype conferred an earlier age at onset than did the common MDM2-SNP309 genotype in the p53 codon R72P GG background, but not in the GC or CC genetic background (Fig. 1). However, the log-rank test showed that the difference between these two genotypes of MDM2-SNP309 was not significant $(p=0.084)$ 

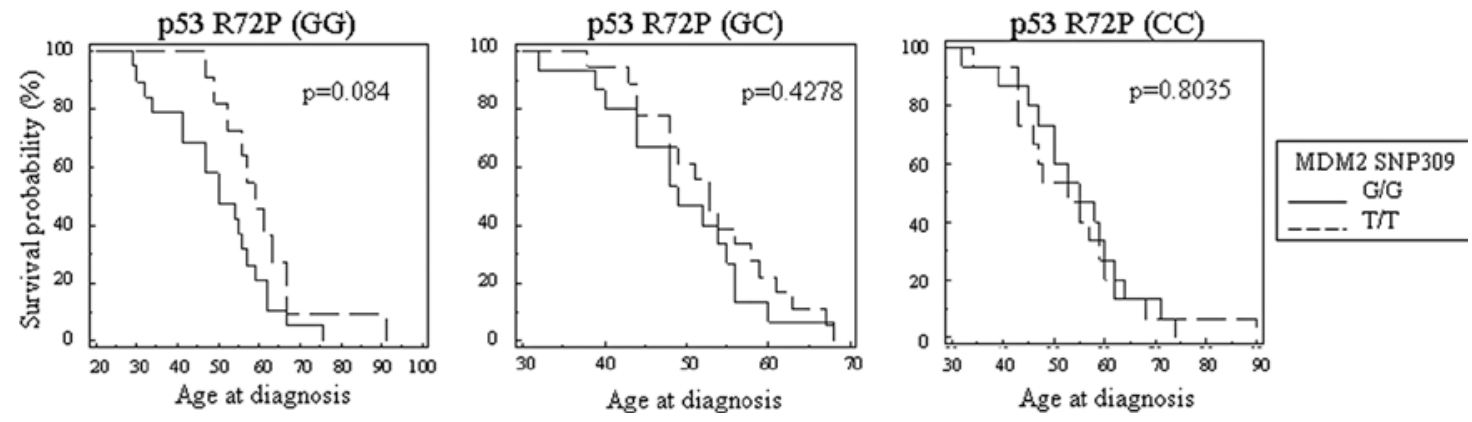

Figure 1. Comparison of the survival curves of MDM2-SNP309 GG and TT carriers after stratification for p53 codon R72P GG (left), GC (middle) and CC (right). The $\mathrm{Y}$ axis represents the cumulative case-free survival rate against age at diagnosis. The statistical significance was determined by the log-rank test.

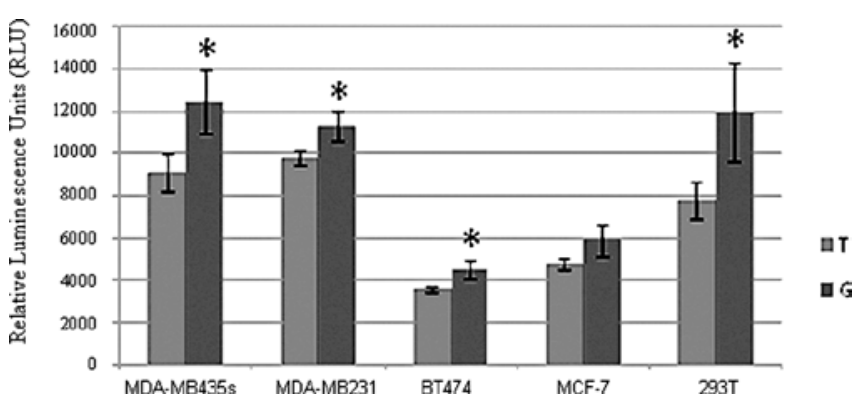

Figure 2. Comparison of the effects of the MDM2-SNP309 G-allele and T-allele on full-length $\mathrm{P} 2$ promoter activity of the MDM2 gene. The results were analyzed by $\mathrm{t}$ test, and the $\mathrm{p}$-values for comparison of the two alleles on promoter activity were $0.0307,0.0294,0.0272,0.065$ and 0.0333 for MDAMB-435s, MDA-MB-231, BT474, MCF-7 and 293T cells, respectively. RLU: relative luminescence units. G, SNP309G-Gluc construct; T, SNP309T-Gluc construct.

even in the background of p53 codon R72P GG genotype. Therefore, the association between MDM2-SNP309 and the age onset of breast cancer in p53 codon R72P GG carriers remains to be addressed in a larger sample size.

MDM2-SNP309 G provides stronger promoter activity than T to the full-length MDM2 P2 promoter. MDM2-SNP309 G has been suggested to increase MDM2 gene expression, whether this effect is cell-specific remains unclear. To investigate the capacity of MDM2-SNP309 to activate the MDM2 gene, the full-length MDM2 P2 promoter containing either G or T was amplified and fused to a Gluc reporter gene (Materials and methods). The reporter gene constructs were separately transfected into several breast cancer cell lines, including MDA-MB435s, MDA-MB231, BT474 and MCF-7. We also transfected these constructs into a non-breast cancer cell line (293T) for comparison. The results showed that the MDM2-SNP309 G allele exhibited a stronger ability than the $\mathrm{T}$ allele to activate the $\mathrm{P} 2$ promoter in all cell lines except in MCF-7 cells, in which the difference was marginally significant ( $\mathrm{p}=0.065)$ (Fig. 2). These data indicate that the $\mathrm{G}$ allele of MDM2-SNP309 generally increases P2 promoter activity, and they imply that the controversial clinical correlation of this genotype with breast cancer is likely associated with a multiplicity of factors.

\section{Discussion}

According to the Taiwan Cancer Registry, breast cancer ranked as the fourth highest type of cancer with respect to mortality among the Taiwanese population during 2001-2008. However, the effects of genetic background on the incidence of breast cancer have not been well-studied. Given that personalized therapeutic strategies are under development, it is important to establish associations between tumor-related genes and cancer risk. Although the germ-line allelic variants of the p53 tumor suppressor gene and the MDM2 oncogene are not always risk factors for various human cancers, their association may be dependent on environmental factors, race or subgroup identity $(11,12,23)$.

In this study, we found that both the heterozygous and homozygous MDM2-SNP309 (TG and GG) genotypes increased the risk of breast cancer (by 1.55- and 1.7-fold, respectively) compared to the wild-type TT genotype in Asian Taiwanese. We also found that p53 codon R72P was not associated with the risk of breast cancer in this population, and this result is consistent with studies based on analyses of large cohorts from multiple countries $(1,11)$, but not with a smallsized sample study (23). The primary limitation of our study is the small sample size. We also found that the distribution of MDM2-SNP309 genotypes in the cancer-free group, but not in the case group, conformed to Hardy-Weinberg equilibrium. This observation is consistent with our previous reports using a smaller cohort (124 cases and 97 controls) to investigate the effects MDM2-SNP309 on breast cancer (12). On the other hand, p53 codon R72P departure from the Hardy-Weinberg law in both the case group and the cancer-free control group. However, this observation should not influence the role of p53 codon R72P on modifying the effect of MDM2-SNP309 on the development of breast cancer.

The association between MDM2-SNP309 and human cancer risk is controversial, and most of the reports have failed to show that carriers of MDM2-SNP309 are more susceptible to cancer (36,39-45). However, environmental factors, hormonal secretion, and inherent or mutant germ-line genetic background may enhance the effects of MDM2-SNP309 on human cancer development $(7,15,25,46-48)$. Compared to previous study (12), here we used a larger sample size for risk evaluation of MDM2-SNP309, and the result remains comparable. Therefore, current data agree that MDM2-SNP309 may be associated with breast cancer risk in selected races and subgroups $(11,16)$. 
In this study, we included a male patient with breast cancer for the statistic analysis. Since the case is insufficient in statistical power this confounding factor has been adjusted for the multivariate logistic regression analysis. In fact, we found that the ORs of MDM2-SNP309 and p53 codon R72P were similar to total cases when only the female groups were calculated (MDM2-SNP309: TG + GG v.s. TT, OR = 1.51, $95 \% \mathrm{CI}=0.959$ to 2.3748 ; $\mathrm{p} 53$ codon $\mathrm{R} 72 \mathrm{P}$ : $\mathrm{GC}+\mathrm{CC}$ v.s. $\mathrm{GG}$, $\mathrm{OR}=1.07,95 \% \mathrm{CI}=0.7048$ to 1.6323 ). Although male breast cancer is rare, the incidence is increasing and the fatality rate of male breast cancer is similar to that of female breast cancer $(49,50)$. Current results suggest that the involvement of male breast cancer in risk evaluation of MDM2-SNP309 or p53 codon R72P genotypes would be meaningful.

To better understand the interaction between MDM2SNP309 and p53 codon R72P, we calculated the ORs of the different MDM2-SNP309 genotypes stratified for p53 codon R72P genotype. We found that in the homozygous p53 codon R72P GG genetic background, both the heterozygous and homozygous MDM2-SNP309 (TG and GG) genotypes conferred a higher risk (OR $=2.4$ and 3.7, respectively) of breast cancer compared to the wild-type MDM2-SNP309 TT genotype. By contrast, the heterozygous and homozygous MDM2-SNP309 genotypes combined with homozygous p53 codon $\mathrm{R} 72 \mathrm{P}$ CC leads to a potentially protective effect $(\mathrm{OR}=0.87$ and 0.66 , respectively) on breast cancer compared to the wild-type MDM2-SNP309 TT genotype. These latter observations are in part consistent with a large cohort study in Europe in which carriers of MDM2-SNP309 GG and p53 codon $\mathrm{R} 72 \mathrm{P} \mathrm{CC}$ had a reduced risk of breast cancer $(\mathrm{OR}=$ $0.68,95 \% \mathrm{CI}=0.43$ to 1.08$)$ (11). In response to stress, the Arg form at $\mathrm{p} 53$ codon $\mathrm{R} 72 \mathrm{P}$ favors cell apoptosis and the Pro form favors cell cycle arrest/DNA repair (17-20). It has been speculated that enhanced risk in carriers of MDM2-SNP309 GG and p53 codon R72P GG is due to the repression of the p53 codon R72P Arg form (apoptosis-prone) by over-expressed MDM2 and subsequent enhanced cell survival after damage. This may lead to an increase in transformed cells that contribute to carcinogenesis. On the other hand, the over-expression of MDM2 by homozygous SNP309 may repress the p53 codon R72P Pro form (DNA repair-prone) and reduce the mutation rate caused by repair errors. The detailed mechanisms, however, remain to be investigated.

In addition to the risk association, homozygous MDM2SNP309 GG genotype showed a trend in accelerating the breast cancer development in the background of p53 codon R72P GG genotype (Arg form). However, since the statistic significance is marginal $(\mathrm{p}=0.084)$, a larger sample size may be required to emphasize this difference. It has been reported that Arg form of p53 codon R72P decreases breast cancer risk in the later onset sporadic cases (14). This may support the hypothesis that the germ-line G allele of MDM2-SNP309 represses the apoptosis-prone p53 codon R72P Arg form or represses the DNA repair-prone p53 codon $\mathrm{R} 72 \mathrm{P}$ Pro form and thereby affects breast cancer development.

Although current data suggest that the effect of MDM2SNP309 on the formation of breast cancer is influenced by the inherent $\mathrm{p} 53$ codon $\mathrm{R} 72 \mathrm{P}$ genetic background, the results are inferred from a small sample. The insufficient power makes it difficult to generalize the statistical analysis. However, a recent international study based on 3,749 breast cancer cases showed that among them, only 26 cases harbored both the homozygous MDM2-SNP309 GG genotype and the p53 codon R72P CC genotype (0.69\%) (33). According to the case data presented in Table III, 15 of the 226 cases were carriers of both MDM2-SNP309 GG and p53 codon R72P CC. This ratio is $6.6 \%$, and it is very similar to that measured for the cancer-free healthy controls. Although a larger sample is required to further address our result, the different allelic frequency of MDM2-SNP309 and p53 codon R72P among selected ethnicities and subgroups agrees with the significance of this factor $(12,16,22,33)$.

Given that the clinical correlation of MDM2-SNP309 genotype with human cancers remains controversial, it is essential to re-evaluate the role of MDM2-SNP309 on the ablation of $\mathrm{P} 2$ promoter activity of the MDM2 gene. Although Bond et al have established reporter gene constructs harboring tandem repeats of the Sp1 binding sequence with either the $\mathrm{T}$ or $\mathrm{G}$ variant at the corresponding SNP309 site (9), to the best of our knowledge the effect on the full-length $\mathrm{P} 2$ promoter of the MDM2 gene has not been investigated. Using reporter gene constructs with either SNP309G or T located in the P2 promoter, we confirmed that the activity of the G-containing promoter is higher than that of the T-containing promoter in five different human cell lines. This difference is only a little weaker in MCF-7 cells, and the p-value remains near 0.05 ( $\mathrm{p}=0.065$ ). Because MDM2-SNP309 only mediates the binding of the Spl general transcription factor, the inherent MDM2-SNP309 genotypes and the MDM2 expression levels of these cell lines should not affect the output of transfected reporter gene constructs. Our results are also consistent with a recent report demonstrating that transgenic mice carrying the MDM2-SNP309G allele exhibit elevated MDM2 levels, repressed p53 levels and increased cancer risk as compared to those carrying the MDM2-SNP309T allele (51). Therefore, the biochemical and molecular functions of MDM2-SNP309 are not likely to be the cause of the clinical controversy on cancer incidence. Genetic background, environmental factors and the geographic distribution of ethnic groups and subgroups may influence the strength of the effect of MDM2-SNP309 on breast cancer development, at least in part.

In summary, our data suggest that the risk allele MDM2SNP309 is associated with the incidence of breast cancer, which becomes greater in the p53 codon R72P GG (Arg form) genetic background. Early age onset of breast cancer in MDM2-SNP309 GG carriers is also enhanced by the p53 codon R72P GG (Arg form) genotype. Given that the $G$ allele of MDM2-SNP309 increases the P2 promoter activity of the MDM2 gene, the association of MDM2-SNP309 with the risk of breast cancer should be multifactorial. Current data suggest that p53 codon R72P may be an effect modifier of MDM2-SNP309 on the development of breast cancer, and this finding may contribute to clinical diagnosis and the design of therapeutic strategies.

\section{Acknowledgements}

This study was supported by the Department of Health in Taipei City Government, (97002-62-091); and the united grant of Cheng-Hsin General Hospital and National Yang-Ming 
University (98F117CY15); and a grant from the Ministry of Education, Aim for the Top University Plan. We also thank Ms. Li-Jian Huang for her help with the collection of blood samples and structured questionnaires.

\section{References}

1. Commonly studied single-nucleotide polymorphisms and breast cancer: results from the Breast Cancer Association Consortium. J Natl Cancer Inst 98: 1382-1396, 2006

2. Eccles D and Tapper W: The influence of common polymorphisms on breast cancer. Cancer Treat Res 155: 15-32, 2010.

3. Speirs V and Shaaban AM: The rising incidence of male breast cancer. Breast Cancer Res Treat 115: 429-430, 2009.

4. Jin S and Levine AJ: The p53 functional circuit. J Cell Sci 114: 4139-4140, 2001.

5. Friedrichs K, Gluba S, Eidtmann H and Jonat W: Overexpression of p53 and prognosis in breast cancer. Cancer 72: 3641-3647, 1993.

6. Thor AD, Moore DH II, Edgerton SM, et al: Accumulation of p53 tumor suppressor gene protein: an independent marker of prognosis in breast cancers. J Natl Cancer Inst 84: 845-855, 1992 .

7. Bond GL, Hirshfield KM, Kirchhoff T, et al: MDM2 SNP309 accelerates tumor formation in a gender-specific and hormonedependent manner. Cancer Res 66: 5104-5110, 2006.

8. Bond GL, Hu W and Levine A: A single nucleotide polymorphism in the MDM2 gene: from a molecular and cellular explanation to clinical effect. Cancer Res 65: 5481-5484, 2005.

9. Bond GL, Hu W, Bond EE, et al: A single nucleotide polymorphism in the MDM2 promoter attenuates the p53 tumor suppressor pathway and accelerates tumor formation in humans. Cell 119: 591-602, 2004.

10. Bond GL and Levine AJ: A single nucleotide polymorphism in the p53 pathway interacts with gender, environmental stresses and tumor genetics to influence cancer in humans. Oncogene 26: 1317-1323, 2007.

11. Schmidt MK, Reincke S, Broeks A, et al: Do MDM2 SNP309 and TP53 R72P interact in breast cancer susceptibility? A large pooled series from the breast cancer association consortium. Cancer Res 67: 9584-9590, 2007.

12. Sun YF, Leu JD, Chen SM, Lin IF and Lee YJ: Results based on 124 cases of breast cancer and 97 controls from Taiwan suggest that the single nucleotide polymorphism (SNP309) in the MDM2 gene promoter is associated with earlier onset and increased risk of breast cancer. BMC Cancer 9: 13, 2009.

13. Wasielewski M, Nagel JH, Brekelmans C, et al: MDM2 SNP309 accelerates familial breast carcinogenesis independently of estrogen signaling. Breast Cancer Res Treat 104: 153-157, 2007.

14. Lum SS, Chua HW, Li H, et al: MDM2 SNP309 G allele increases risk but the $\mathrm{T}$ allele is associated with earlier onset age of sporadic breast cancers in the Chinese population. Carcinogenesis 29: 754-761, 2008.

15. Yarden RI, Friedman E, Metsuyanim S, Olender T, Ben-Asher E and Papa MZ: MDM2 SNP309 accelerates breast and ovarian carcinogenesis in BRCA 1 and BRCA 2 carriers of JewishAshkenazi descent. Breast Cancer Res Treat 111: 497-504, 2008.

16. Economopoulos KP and Sergentanis TN: Differential effects of MDM2 SNP309 polymorphism on breast cancer risk along with race: a meta-analysis. Breast Cancer Res Treat 120: 211-216, 2010.

17. Pietsch EC, Humbey $\mathrm{O}$ and Murphy ME: Polymorphisms in the p53 pathway. Oncogene 25: 1602-1611, 2006.

18. Dumont P, Leu JI, Della Pietra AC III, George DL and Murphy M: The codon 72 polymorphic variants of $\mathrm{p} 53$ have markedly different apoptotic potential. Nat Genet 33: 357-365, 2003.

19. Pim D and Banks L: p53 polymorphic variants at codon 72 exert different effects on cell cycle progression. Int J Cancer 108: 196-199, 2004.

20. Siddique $M$ and Sabapathy K: Trp53-dependent DNA-repair is affected by the codon 72 polymorphism. Oncogene 25: 3489-3500, 2006.

21. Siddique MM, Balram C, Fiszer-Maliszewska L, et al: Evidence for selective expression of the p53 codon 72 polymorphs: implications in cancer development. Cancer Epidemiol Biomarkers Prev 14: 2245-2252, 2005.
22. Zhang Z, Wang M, Wu D, et al: P53 codon 72 polymorphism contributes to breast cancer risk: a meta-analysis based on 39 case-control studies. Breast Cancer Res Treat 120: 509-517, 2010.

23. Papadakis EN, Dokianakis DN and Spandidos DA: p53 codon 72 polymorphism as a risk factor in the development of breast cancer. Mol Cell Biol Res Commun 3: 389-392, 2000.

24. Freedman DA and Levine AJ: Regulation of the p53 protein by the MDM2 oncoprotein - Thirty-eighth G.H.A. Clowes Memorial Award Lecture. Cancer Res 59: 1-7, 1999.

25. Dharel N, Kato N, Muroyama R, et al: MDM2 promoter SNP309 is associated with the risk of hepatocellular carcinoma in patients with chronic hepatitis C. Clin Cancer Res 12: 4867-4871, 2006.

26. Grochola LF, Muller TH, Bond GL, Taubert H, Udelnow A and Wurl P: MDM2 SNP309 associates with accelerated pancreatic adenocarcinoma formation. Pancreas 39: 76-80, 2010.

27. Idbaih A, Boisselier B, Marie Y, et al: Influence of MDM2 SNP309 alone or in combination with the TP53 R72P polymorphism in oligodendroglial tumors. Brain Res 1198: 16-20, 2008.

28. Bougeard G, Baert-Desurmont S, Tournier I, et al: Impact of the MDM2 SNP309 and p53 Arg72Pro polymorphism on age of tumour onset in Li-Fraumeni syndrome. J Med Genet 43 . 531-533, 2006

29. Ashton KA, Proietto A, Otton G, et al: Polymorphisms in TP53 and MDM2 combined are associated with high grade endometrial cancer. Gynecol Oncol 113: 109-114, 2009.

30. Talseth BA, Meldrum C, Suchy J, Kurzawski G, Lubinski J and Scott RJ: MDM2 SNP309 T>G alone or in combination with the TP53 R72P polymorphism does not appear to influence disease expression and age of diagnosis of colorectal cancer in HNPCC patients. Int J Cancer 120: 563-565, 2007.

31. Toffoli G, Biason P, Russo A, et al: Effect of TP53 Arg72Pro and MDM2 SNP309 polymorphisms on the risk of high-grade osteosarcoma development and survival. Clin Cancer Res 15: 3550-3556, 2009.

32. Horikawa Y, Nadaoka J, Saito M, et al: Clinical implications of the MDM2 SNP309 and p53 Arg72Pro polymorphisms in transitional cell carcinoma of the bladder. Oncol Rep 20: 49-55, 2008.

33. Schmidt MK, Tommiska J, Broeks A, et al: Combined effects of single nucleotide polymorphisms TP53 R72P and MDM2 SNP309, and p53 expression on survival of breast cancer patients. Breast Cancer Res 11: R89, 2009.

34. Lang A, Palmeback Wegman $P$ and Wingren $S$ : The significance of MDM2 SNP309 and p53 Arg72Pro in young women with breast cancer. Oncol Rep 22: 575-579, 2009.

35. Singh V, Rastogi N, Mathur N, Singh K and Singh MP: Association of polymorphism in MDM-2 and p53 genes with breast cancer risk in Indian women. Ann Epidemiol 18: 48-57, 2008.

36. Cox DG, Deer D, Guo Q, et al: The p53 Arg72Pro and MDM2-309 polymorphisms and risk of breast cancer in the nurses' health studies. Cancer Causes Control 18: 621-625, 2007.

37. Walsh CS, Miller CW, Karlan BY and Koeffler HP: Association between a functional single nucleotide polymorphism in the MDM2 gene and sporadic endometrial cancer risk. Gynecol Oncol 104: 660-664, 2007.

38. Xu Y, Yao L, Zhao A, et al: Effect of 553 codon 72 genotype on breast cancer survival depends on p53 gene status. Int J Cancer 122: 2761-2766, 2008.

39. Pine SR, Mechanic LE, Bowman ED, et al: MDM2 SNP309 and SNP354 are not associated with lung cancer risk. Cancer Epidemiol Biomarkers Prev 15: 1559-1561, 2006.

40. Millikan RC, Heard K, Winkel S, et al: No association between the MDM2 -309 T/G promoter polymorphism and breast cancer in African-Americans or Whites. Cancer Epidemiol Biomarkers Prev 15: 175-177, 2006.

41. Krekac D, Brozkova K, Knoflickova D, et al: MDM2SNP309 does not associate with elevated MDM2 protein expression or breast cancer risk. Oncology 74: 84-87, 2008.

42. Hu Z, Jin G, Wang L, Chen F, Wang X and Shen H: MDM2 promoter polymorphism SNP309 contributes to tumor susceptibility: evidence from 21 case-control studies. Cancer Epidemiol Biomarkers Prev 16: 2717-2723, 2007.

43. Copson ER, White HE, Blaydes JP, Robinson DO, Johnson PW and Eccles DM: Influence of the MDM2 single nucleotide polymorphism SNP309 on tumour development in BRCA1 mutation carriers. BMC Cancer 6: 80, 2006.

44. Campbell IG, Eccles DM and Choong DY: No association of the MDM2 SNP309 polymorphism with risk of breast or ovarian cancer. Cancer Lett 240: 195-197, 2006. 
45. Ma H, Hu Z, Zhai X, et al: Polymorphisms in the MDM2 promoter and risk of breast cancer: a case-control analysis in a Chinese population. Cancer Lett 240: 261-267, 2006.

46. Boersma BJ, Howe TM, Goodman JE, et al: Association of breast cancer outcome with status of p53 and MDM2 SNP309. J Natl Cancer Inst 98: 911-919, 2006.

47. Menin C, Scaini MC, De Salvo GL, et al: Association between MDM2-SNP309 and age at colorectal cancer diagnosis according to p53 mutation status. J Natl Cancer Inst 98: 285-288, 2006.

48. Leu JD, Lin IF, Sun YF, Chen SM, Liu CC and Lee YJ: Association between MDM2-SNP309 and hepatocellular carcinoma in Taiwanese population. World J Gastroenterol 15: 5592-5597, 2009.
49. Ottini L, Palli D, Rizzo S, Federico M, Bazan V and Russo A: Male breast cancer. Crit Rev Oncol Hematol 73: 141-155, 2010.

50. Anderson WF, Jatoi I, Tse J and Rosenberg PS: Male breast cancer: a population-based comparison with female breast cancer. J Clin Oncol 28: 232-239, 2010.

51. Post SM, Quintas-Cardama A, Pant V, et al: A high-frequency regulatory polymorphism in the $\mathrm{p} 53$ pathway accelerates tumor development. Cancer Cell 18: 220-230, 2010. 